\title{
A recalcitrant foreign body
}

\author{
Ashley Gillson MD¹, Alain Tremblay MDCM²
}

\begin{abstract}
A 54-year-old-woman with well-controlled mild intermittent asthma presented with a persistent cough productive of purulent sputum. She had no symptoms of dysphagia or gastroesophageal reflux disease. She had a remote 5-pack-year smoking history and previously lived in Lake of the Woods, Ontario, where her and her husband owned and operated a tree planting company for many years. There were no risk factors for tuberculosis nor travel out of country. Computed tomography of the chest (Figure 1A) revealed an obstructing calcified lesion within the anterior segment of the right upper lobe and calcified lymphadenopathy present in the right paratracheal region. Additional calcified granulomas were noted within the right middle and lower lobes. Flexible bronchoscopy confirmed the presence of an obstructing lesion within the anterior segment of the right upper lobe (Figure 1B). Attempts were made to extract the lesion via flexible bronchoscopy, but because these were unsuccessful, a rigid bronchoscopy was performed to remove the lesion (Figure 1C). Pathological examination was consistent with a broncholith, hypothesized to be related to blastomycosis infection given her occupation and residence in an endemic area, although cultures and fungal serology were negative.
\end{abstract}

\section{KEY LEARNING POINTS}

- Although rare, broncholithiasis should be included in the differential diagnosis of a suspected foreign body. Additional evidence of previous granulomatous inflammation and calcification may be supportive.
- Other considerations for a calcified endobronchial lesion include the following: chronic endobronchial infection; carcinoid tumours; hamartoma; amyloidosis; tracheobronchoplathia osteochondroplastica; osteomas; osteosarcomas; and chondromas and chondrosarcomas. All of these are rare.

- Symptoms from broncholithiasis may include hemoptysis, persistent cough and recurrent pneumonia.

- Treatment should be offered in symptomatic patients.

- Broncholiths can occasionally be safely removed by flexible bronchoscopic techniques, but in more difficult cases, rigid bronchoscopy or surgical bronchotomy may be required.

\section{REFERENCES}

1. Anwer M, Venkatram S. Broncholithiasis: Incidental finding during bronchoscopy - case reports and review of the literature. J Bronchol Intervent Pulmonol 2011;18:181-3.

2. Cerfolio RJ, Bryant AS, Maniscalco L. Rigid bronchoscopy and surgical resection for broncholithiasis and calcified mediastinal lymph nodes.J Thorac Cardiovasc Surg 2008;136:186-90.

3. Seo JB, Song KS, Lee JS, et al. Broncholithiasis: Review of the causes with radiologic-pathologic correlation. Radiographics 2002;S199-213.

4. Choptiany M, Wiebe L, Limerick B, et al. Risk factors for acquisition of endemic blastomycosis. Can J Infect Dis Med Microbiol 2009;20:117-21.
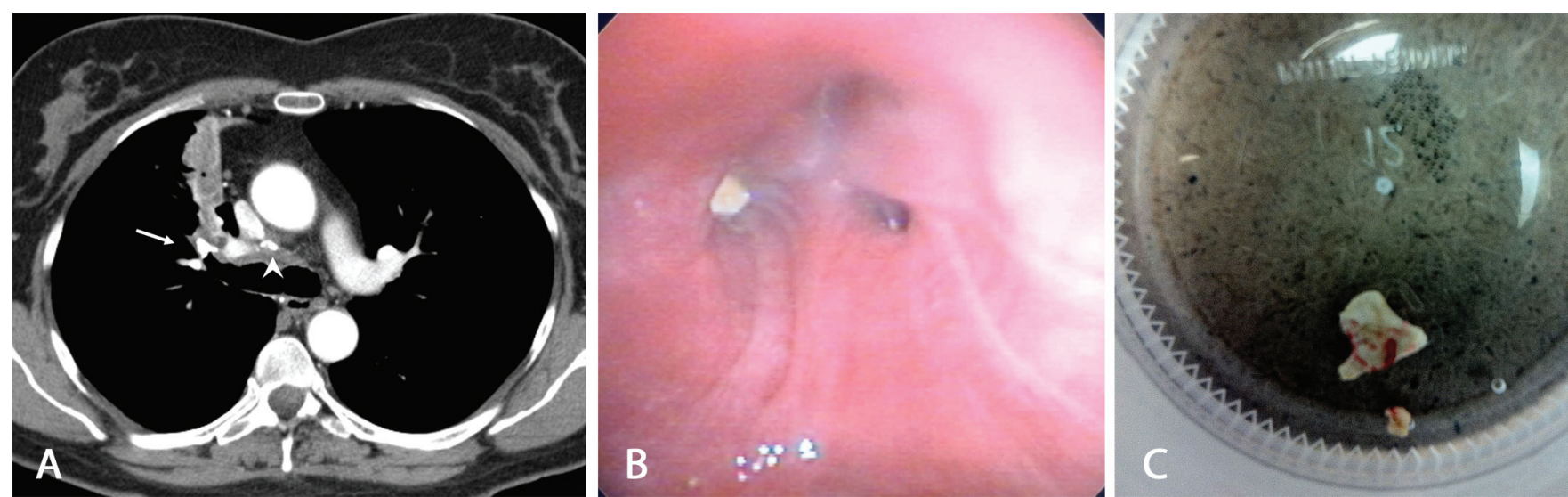

Figure 1) A A calcified obstructing lesion (white arrow) seen on computed tomography within the anterior segment of the right upper lobe with calcified mediastinal lymphadenopathy (white arrowhead). Mucous plugging and atelectasis is seen distal to the lesion. B Image from flexible bronchoscopy confirming the obstructing lesion within the anterior segment of the right upper lobe. C Gross specimen of the lesion (10 mm largest diameter) extracted from the right upper lobe, placed in a plastic specimen container

The 'Images in Respiratory Medicine' section of the Canadian Respiratory Journal aims to highlight the importance of visual interpretation, whether physiological, radiological, bronchoscopic, surgical/thorascopic or histological, in the diagnosis of chest diseases. Submissions should exemplify a classic, particularly dramatic or intriguing presentation of a disease while offering an important educational message to the reader (insightful diagnostic pearls or differential diagnosis, etc). This section is not intended to be a vehicle for publication of case reports (see the Clinical-Pathologic-Conferences for case-based leaning series).

${ }^{1}$ University of Alberta, Edmonton; ${ }^{2}$ Department of Medicine, University of Calgary, Calgary, Alberta

Corresponence: Dr Alain Tremblay, Department of Medicine, University of Calgary, 3330 Hospital Drive Northwest, Calgary Alberta T2N 4N1.

Telephone 403-210-3866, fax 403-944-1577, e-mail alain.tremblay@ucalgary.ca 


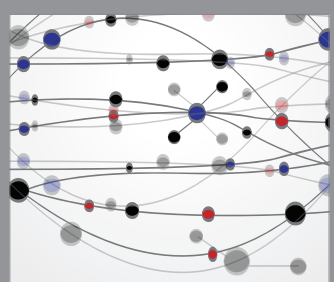

The Scientific World Journal
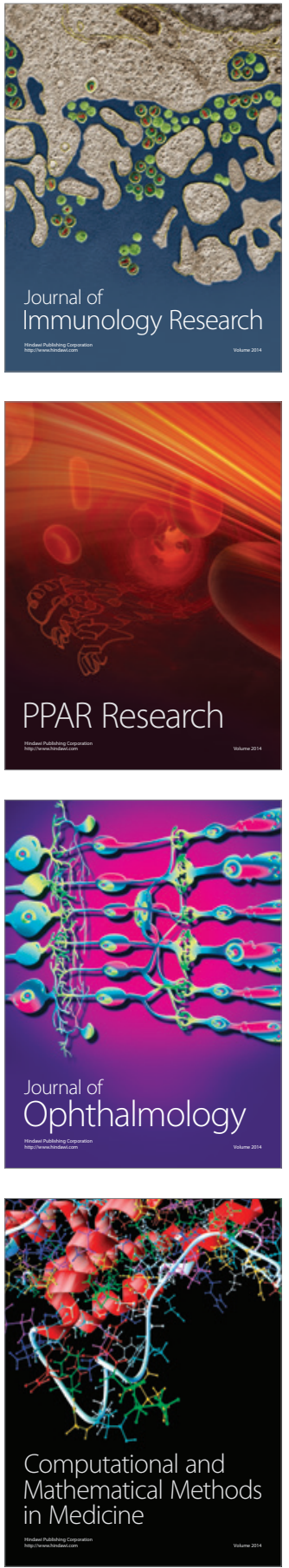

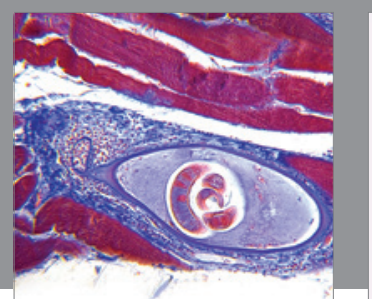

Gastroenterology Research and Practice

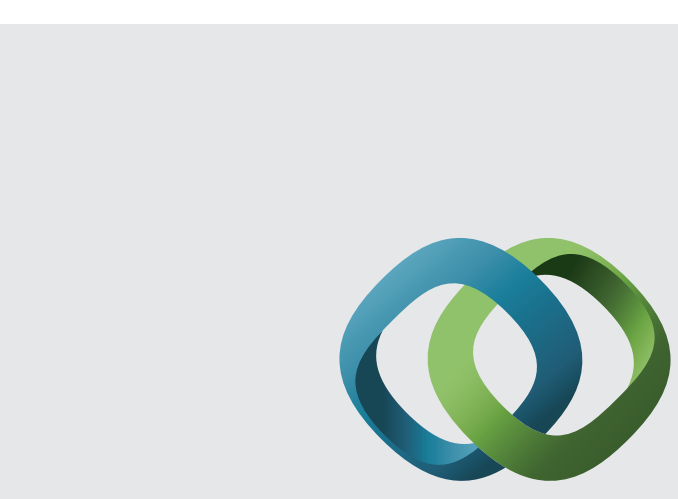

\section{Hindawi}

Submit your manuscripts at

http://www.hindawi.com
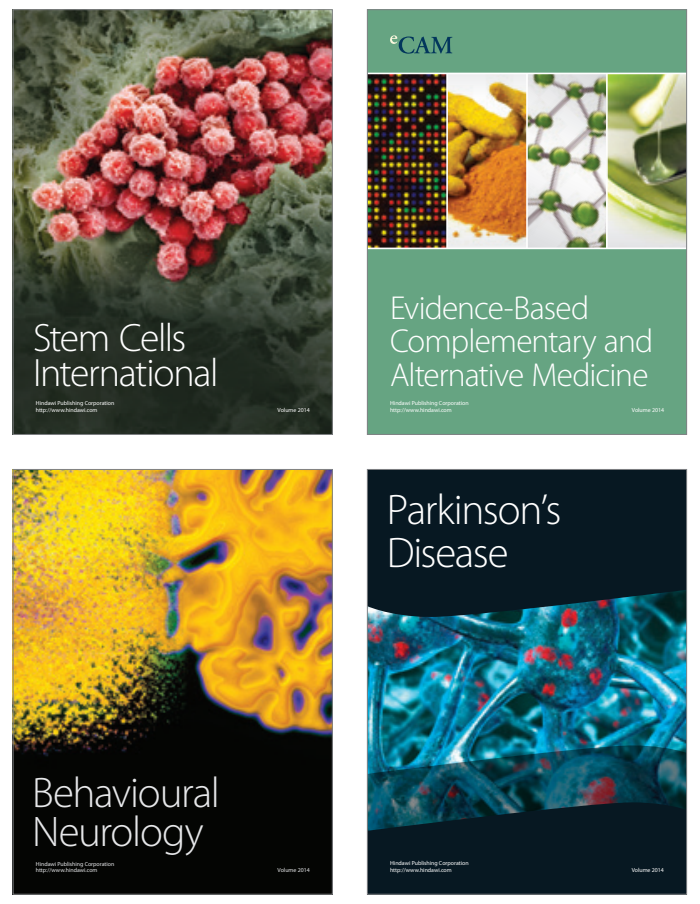
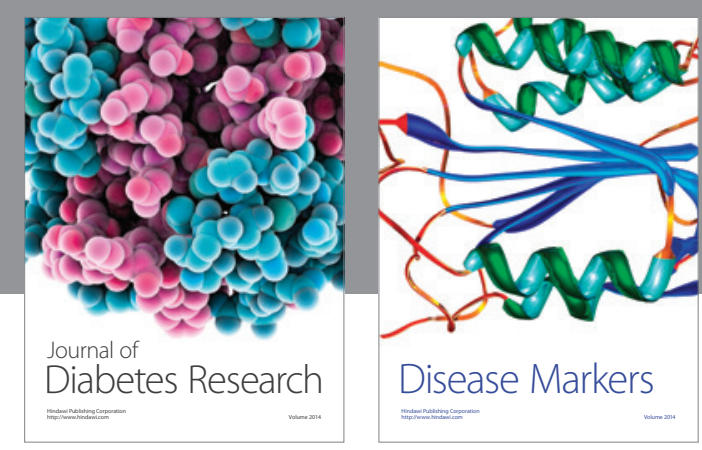

Disease Markers
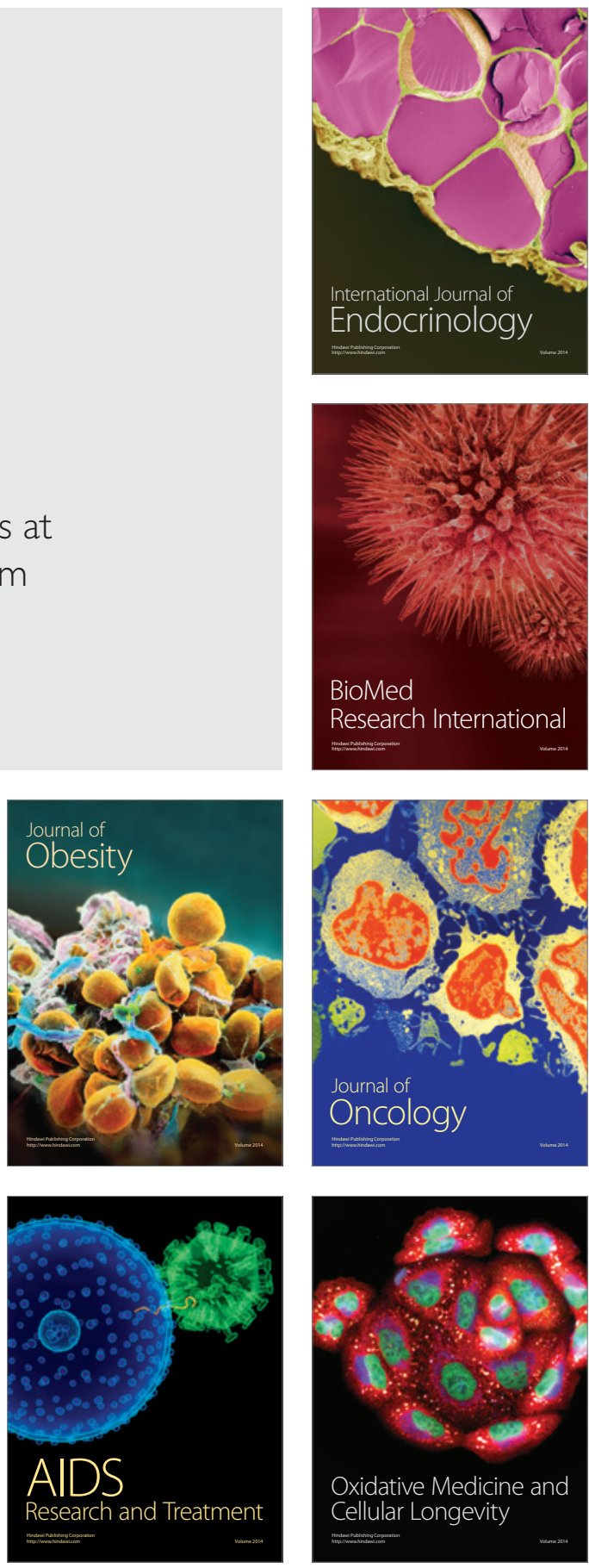